\title{
Single-cell transcriptional profile of ACE2 in healthy and failing human hearts
}

\author{
Yitian Zhou ${ }^{1,2 \dagger}$, Yongfa Huang ${ }^{2 \dagger}$, Xiaomin Song ${ }^{1 \dagger}$, Xiaoxiao Guo $^{2 \dagger}$, Junling Pang ${ }^{1}$, Jing Wang ${ }^{1 *}$, \\ Shuyang Zhang ${ }^{2 *} \&$ Chen Wang ${ }^{3}$ \\ ${ }^{1}$ State Key Laboratory of Medical Molecular Biology, Institute of Basic Medical Sciences, Chinese Academy of Medical Sciences, Department \\ of Pathophysiology, Peking Union Medical College, Beijing 100730, China; \\ ${ }^{2}$ Department of Cardiology, Peking Union Medical College Hospital, Peking Union Medical College and Chinese Academy of Medical \\ Sciences, Beijing 100730, China; \\ ${ }^{3}$ State Key Laboratory of Medical Molecular Biology, Institute of Basic Medical Sciences, Chinese Academy of Medical Sciences, Department \\ of Physiology, Peking Union Medical College, Beijing 100730, China
}

Received May 20, 2020; accepted August 16, 2020; published online September 1, 2020

Citation: Zhou, Y., Huang, Y., Song, X., Guo, X., Pang, J., Wang, J., Zhang, S., and Wang, C. (2021). Single-cell transcriptional profile of ACE2 in healthy and failing human hearts. Sci China Life Sci 64, 652-655. https://doi.org/10.1007/s11427-020-1787-5

Dear Editor,

The coronavirus disease 2019 (COVID-19) pandemic, which is caused by SARS-CoV-2, has gained serious attention from medical practitioners around the world in the past few months. Approximately $20 \%$ of critically ill COVID-19 patients were reported to have suffered myocardial injury. The specific mechanism of this pathology requires further investigation (Yang et al., 2020). It was reported that SARSCoV-2 invades alveolar epithelial cells via the angiotensin converting enzyme (ACE2) cell receptor in the lungs, but whether cardiomyocytes can be directly infected with SARSCoV-2 via ACE2 remains controversial (Zhou et al., 2020). The rapid development of single-cell RNA sequencing (scRNA-seq) in recent years has enabled us to analyze the transcriptome with a single-cell resolution. In this study, we analyzed the scRNA-seq data from human hearts in the Gene Expression Omnibus public database to investigate the expression profile of ACE2 and the expression profiles of other genes in different types of human heart cells.

$\dagger$ Contributed equally to this work

*Corresponding authors (Jing Wang, email: wangjing@ibms.pumc.edu.cn; Shuyang

Zhang, email: shuyangzhang103@163.com)
Briefly, the scRNA-seq data from the heart samples of 14 healthy adults and four heart failure patients (GSE109816, GSE121893), as well as those from 20 fetal hearts (GSE106118), were included in the analysis. The Seurat $\mathrm{R}$ package (v.3.1.2) was used to perform data integration and clustering analysis to approximately 14,000 single cells (Stuart et al., 2019). The clusters were annotated based on canonical cell-type marker genes (Han et al., 2018; Volz et al., 2015). The main cell types of the heart, including cardiomyocytes and cardiac fibroblasts, were identified in both the hearts of adult humans and the hearts of human embryos (Figure 1A, B, and H). Consistent with the results of previous studies analyzing the expression levels of ACE2 in cardiomyocytes (Xu H et al., 2020), we detected that about $10 \%$ of cardiomyocytes had ACE2 expression (Figure 1C and D). The expression levels of ACE2 in the atria and ventricles were not significantly different (Figure $1 \mathrm{~F}$ ).

More interestingly, we found that nearly $30 \%$ of pericytes expressed ACE2 and that the expression level of ACE2 in pericytes was much higher than that in cardiomyocytes (Figure 1E). Our results are consistent with the results of a previous study that suggested ACE2 is highly expressed in pericytes in the heart tissue of humans (Chen et al., 2020). Furthermore, both the expression level and percentage of 

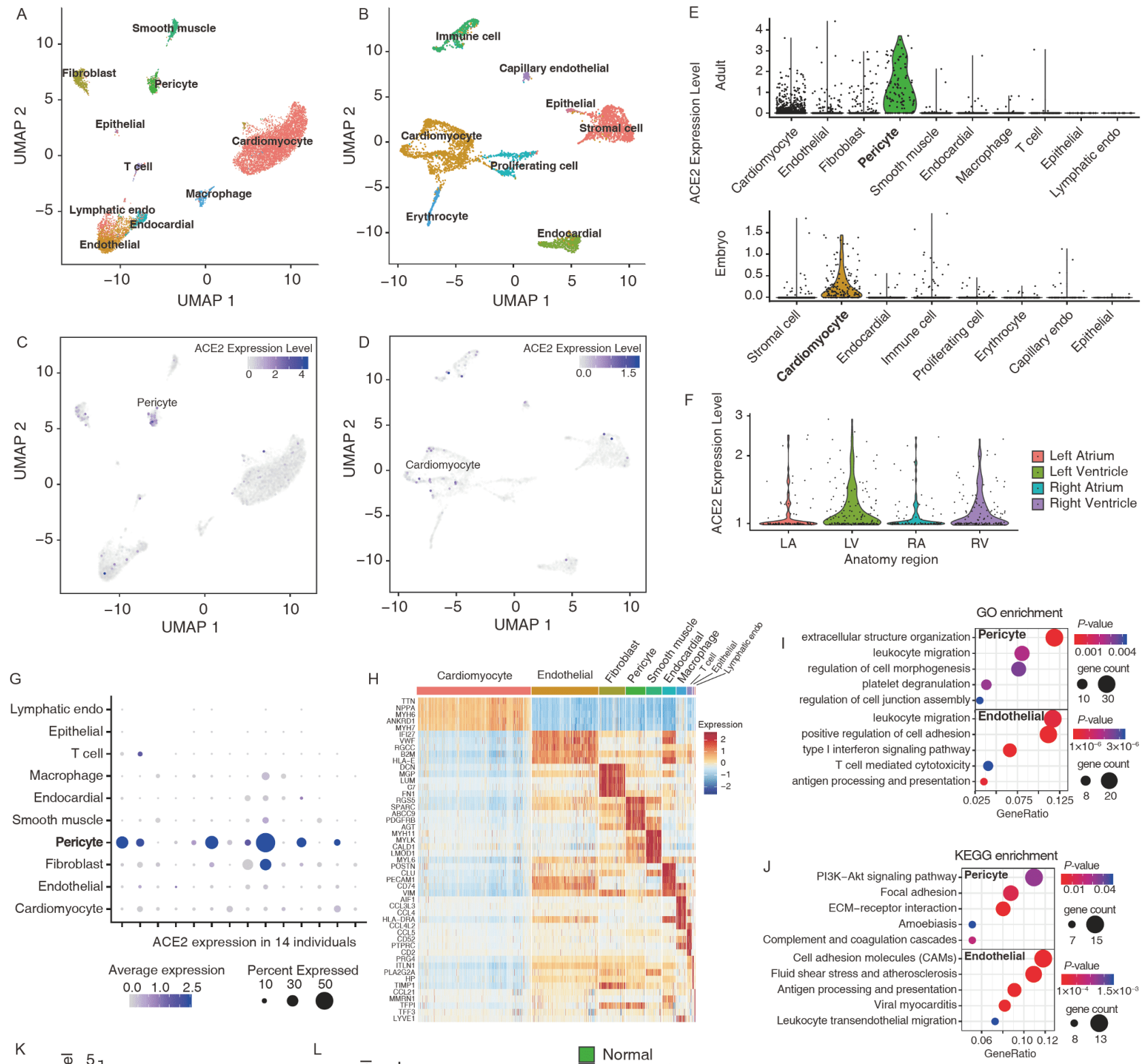

$\mathrm{K}$

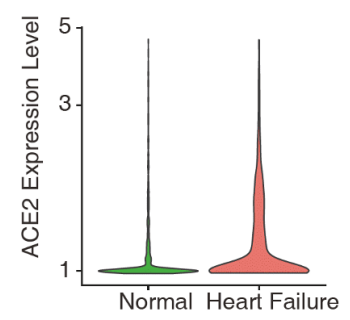

L

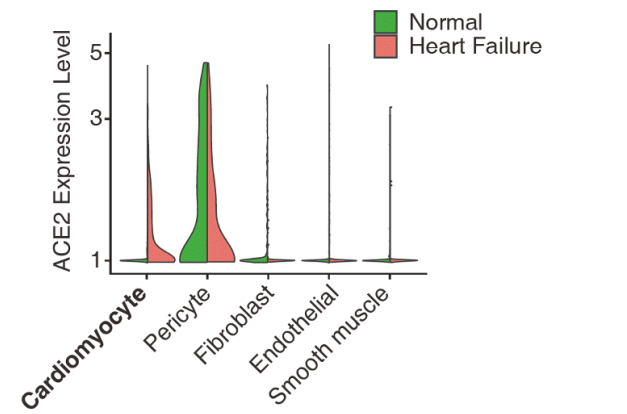

Figure 1 Characterization of the single-cell RNA expression transcriptional profile of ACE2 in the heart. A and B, Uniform manifold approximation and projection (UMAP) dimension reduction showing the results of unsupervised clustering for all filtered cells from the heart of an adult human (A), $n=10,314$, and from the heart of a human embryo (B), $n=4,617$. The cell types corresponding to each cluster are highlighted with their names labeled. C and $\mathrm{D}$, Expression patterns of ACE2 in single cells from the heart of an adult human (C) and from the heart of a human embryo (D) under UMAP dimension reduction. Each dot represents a cell with a color indicating the normalized level of ACE2 expression. E, Violin plots showing the expression profiles of ACE2 in different cell types of the hearts of both human adults and embryos. F, Violin plot of normalized ACE2 expression levels in different anatomical regions of the heart in the human embryo. G, Expression profiles of ACE2 in different cell types of hearts from 14 adult human individuals. H, Heatmap showing the expression levels of the top 5 marker genes in each cluster from the hearts of adult humans. I and J, GO (I) and KEGG (J) enrichment using marker genes (log-fold change $>0.5$ ) of pericyte and endothelial cells from the heart of an adult human. For each group, five of the top 20 items are presented. $\mathrm{K}$, Expression levels of ACE2 in normal individuals $(n=14)$ and heart failure patients $(n=4)$. L, ACE2 expression levels in different cell types of hearts for 14 adult human individuals and four heart failure patients. 
pericytes were markedly higher in six of the adults than they were in the other eight adults (Figure 1G). However, whether ACE2 expression is heterogeneous in the general population requires further study. Since pericytes express ACE2 the highest among other cells and endothelial cells are closely related to pericytes in structure and function (Chiaverina et al., 2019; Stallcup, 2018), we next used markers of pericytes and endothelial cells to perform Gene Ontology (GO) and Kyoto Encyclopedia of Genes and Genomes (KEGG) enrichment analysis using the ClusterProfiler $\mathrm{R}$ package (v.3.15.2). As shown in Figure 1I and J, pericytes feature signaling pathways related to extracellular structure interactions and complement and coagulation cascades, while endothelial cells feature interferon and antigen presentation signaling pathways. These findings illustrate the importance of the roles that pericytes and endothelial cells play and their potential interactions, such as the joint regulation of leukocyte migration and cell adhesion.

Our results suggest that ACE2 transcripts in the heart are mainly concentrated in pericytes and not in cardiomyocytes. Moreover, the heart biopsy sample of a COVID-19 patient who died of cardiac arrest showed only a few interstitial mononuclear inflammatory infiltrates (Xu Z et al., 2020). Both of these findings suggest that the virus attacks mainly pericytes rather than cardiomyocytes in the healthy human heart and that it does so via ACE2. The classic paradigm of viral myocarditis cannot be directly applied to myocardial injury in the general population. Pericytes are contractile vascular mural cells that closely interact with endothelial cells, and together, these cells play an important role in regulating the vascular tone of coronary microcirculation. Similarly, disorders of pericytes alone can also lead to microvascular constriction, as it is in a no-reflow phenomenon (O'Farrell and Attwell, 2014). Appropriately, we propose that SARS-CoV-2 might attack pericytes via ACE2 and result in myocardial disorder in some patients.

A recent study suggested an incidence of thrombotic events including venous and arterial thrombosis of $31 \%$ in COVID-19 patients (Klok et al., 2020). Most studies indicate that thrombosis is closely related to endothelial dysfunction (Escher et al., 2020; Sardu et al., 2020), which is a finding that is in agreement with our finding that approximately $3 \%$ endothelial cells show expression of ACE2 (Figure 1E and $\mathrm{G})$. Interestingly, our results show that the expression profile of pericytes features pathways related to coagulation cascades, indicating potential participation of pericytes in thrombotic events. Further studies should be performed to elucidate the roles of endothelial cells and pericytes in venous and arterial thrombosis in the pathogenesis of COVID19.

In addition, we found that $10 \%$ of cardiac fibroblasts express ACE2 (Figure 1G). Cardiac fibroblasts secrete collagens and other extracellular matrix proteins to promote fibrosis and diastolic dysfunction (Travers et al., 2016). Furthermore, activated fibroblasts may also regulate cardiomyocyte survival and hypertrophy by secreting growth factors or miRNA-containing exosomes, which contribute to myocardial injury (Humeres and Frangogiannis, 2019). We propose that dysregulation of cardiac fibroblasts might cause worsening of myocardial injury in heart disease patients with COVID-19.

Multiple mechanisms can lead to myocardial injury. The most suspected ones in COVID-19 are hypoxemia, hypotension, inflammatory and stress responses, and pre-existing heart diseases (Thygesen et al., 2019). Disseminated intravascular coagulation was observed in approximately $70 \%$ of COVID-19 non-survivors, and it is reasonable to suspect that thrombosis can occur within small tributaries of the coronary arteries and result in myocardial ischemia (Tang et al., 2020). The aforementioned pericyte-related microvascular disorder might also contribute to ischemia and result in myocardial injury. As the expression level of ACE2 is relatively low in cardiomyocytes, the presence of viral myocarditis in COVID-19 patients is highly suspected. In addition, only subclinical diastolic impairment without systolic involvement was observed in patients infected with SARS-CoV, which is a close relative to SARS-CoV-2. These results are inconsistent with the clinical characteristics of viral myocarditis ( $\mathrm{Li}$ et al., 2003). Therefore, it is possible that the cardiac injury shown in COVID-19 is initiated from an attack on pericytes via ACE2.

Cardiovascular disease patients are more likely to be infected by SARS-CoV-2 than those without cardiovascular disease, which may aggravate the disease progression (Chen et al., 2020). Therefore, we further analyzed the expression of ACE2 in heart failure patients. Our results showed that ACE2 expression was significantly increased in patients with heart failure compared to healthy individuals (Figure $1 \mathrm{~K}$, $P<0.001$, Wilcoxon test). To explore the potential mechanisms contributing to this finding, we examined the expression of ACE2 in the different cells of the heart for heart samples from healthy individuals and heart failure patients and compared the results between these two groups. We found that the expression of ACE2 in cardiomyocytes was significantly increased compared to the other cells in the heart failure patients (Figure 1L). These results suggest that the cardiomyocytes of heart failure patients may be directly attacked by the virus and that this may contribute to the development of myocardial injury and severe conditions.

A recent study showed that ACE2 is highly expressed in pericytes in the heart (Chen et al., 2020). Our results were produced from analyses that included even more heart samples, and our results further confirmed that ACE2 is highly expressed in pericytes in the heart. We speculate that SARS-CoV-2 mainly attacks pericytes via ACE2, which leads to coronary microvascular disorder and myocardial 
injury in COVID-19 patients. Moreover, our results also indicated that the expression of ACE2 in pericytes for six adults was higher than that for the other eight adults, which implies that the heterogeneity of ACE2 expression in the hearts of those in the general population is worth further exploration. In addition, consistent with the results of a previous study, we found that ACE2 expression is increased in heart failure patients (Chen et al., 2020). More importantly, we further showed that the expression of ACE2 in the cardiomyocytes of heart failure patients is significantly higher than that in other heart cells of these patients. This finding suggests that heart failure patients infected by the SARS-CoV-2 virus may suffer viral myocarditis and severe myocardial injury by direct attack on their cardiomyocytes. These results imply that previous heart disease is closely related to whether people infected by the SARS-CoV-2 virus will develop myocardial damage.

In conclusion, the expression of ACE2 in cardiomyocytes is significantly increased in heart failure patients compared to healthy individuals. Heart failure patients may have a high risk for developing viral myocarditis and severe myocardial injury due to cardiomyocyte dysfunction.

\section{Compliance and ethics The author(s) declare that they have no conflict} of interest.

\section{Acknowledgements This work was financially supported by Chinese Academy of Medical Sciences Innovation Fund for Medical Sciences (2018- 12M-1-001) and Consulting Research Project of Chinese Academy of En- gineering (2020-KYGG-01-05).}

\section{References}

Chen, L., Li, X., Chen, M., Feng, Y., and Xiong, C. (2020). The ACE2 expression in human heart indicates new potential mechanism of heart injury among patients infected with SARS-CoV-2. Cardiovasc Res 116, $1097-1100$.

Chiaverina, G., di Blasio, L., Monica, V., Accardo, M., Palmiero, M., Peracino, B., Vara-Messler, M., Puliafito, A., and Primo, L. (2019). Dynamic interplay between pericytes and endothelial cells during sprouting angiogenesis. Cells 8, 1109.

Escher, R., Breakey, N., and Lämmle, B. (2020). Severe COVID-19 infection associated with endothelial activation. Thromb Res 190, 62.

Han, X., Wang, R., Zhou, Y., Fei, L., Sun, H., Lai, S., Saadatpour, A., Zhou, Z., Chen, H., Ye, F., et al. (2018). Mapping the mouse cell atlas by microwell-seq. Cell 172, 1091-1107.e17.

Humeres, C., and Frangogiannis, N.G. (2019). Fibroblasts in the infarcted, remodeling, and failing heart. JACC Basic Transl Sci 4, 449-467.

Klok, F.A., Kruip, M.J.H.A., van der Meer, N.J.M., Arbous, M.S., Gommers, D.A.M.P.J., Kant, K.M., Kaptein, F.H.J., van Paassen, J., Stals, M.A.M., Huisman, M.V., et al. (2020). Incidence of thrombotic complications in critically ill ICU patients with COVID-19. Thromb Res 191, 145-147.

Li, S.S., Cheng, C., Fu, C., Chan, Y., Lee, M., Chan, J.W., and Yiu, S. (2003). Left ventricular performance in patients with severe acute respiratory syndrome. Circulation $108,1798-1803$.

O'Farrell, F.M., and Attwell, D. (2014). A role for pericytes in coronary noreflow. Nat Rev Cardiol 11, 427-432.

Sardu, C., Gambardella, J., Morelli, M.B., Wang, X., Marfella, R., and Santulli, G. (2020). Hypertension, thrombosis, kidney failure, and diabetes: is COVID-19 an endothelial disease? A comprehensive evaluation of clinical and basic evidence. J Clin Med 9, 1417.

Stallcup, W.B. (2018). The NG2 proteoglycan in pericyte biology. Adv Exp Med Biol 1109, 5-19.

Stuart, T., Butler, A., Hoffman, P., Hafemeister, C., Papalexi, E., Mauck Iii, W.M., Hao, Y., Stoeckius, M., Smibert, P., and Satija, R. (2019). Comprehensive integration of single-cell data. Cell 177, 1888-1902. e21.

Tang, N., Li, D., Wang, X., and Sun, Z. (2020). Abnormal coagulation parameters are associated with poor prognosis in patients with novel coronavirus pneumonia. J Thromb Haemost 18, 844-847.

Thygesen, K., Alpert, J.S., Jaffe, A.S., Chaitman, B.R., Bax, J.J., Morrow, D.A., White, H.D., Thygesen, K., Alpert, J.S., Jaffe, A.S., et al. (2019). Fourth universal definition of myocardial infarction (2018). Eur Heart J 40, 237-269.

Travers, J.G., Kamal, F.A., Robbins, J., Yutzey, K.E., and Blaxall, B.C. (2016). Cardiac fibrosis. Circ Res 118, 1021-1040.

Volz, K.S., Jacobs, A.H., Chen, H.I., Poduri, A., McKay, A.S., Riordan, D. P., Kofler, N., Kitajewski, J., Weissman, I., and Red-Horse, K. (2015). Pericytes are progenitors for coronary artery smooth muscle. eLife 4, e10036.

Xu, H., Zhong, L., Deng, J., Peng, J., Dan, H., Zeng, X., Li, T., and Chen, Q. (2020). High expression of ACE2 receptor of 2019-nCoV on the epithelial cells of oral mucosa. Int J Oral Sci 12, 8.

Xu, Z., Shi, L., Wang, Y., Zhang, J., Huang, L., Zhang, C., Liu, S., Zhao, P., Liu, H., Zhu, L., et al. (2020). Pathological findings of COVID-19 associated with acute respiratory distress syndrome. Lancet Respir Med $8,420-422$.

Yang, X., Yu, Y., Xu, J., Shu, H., Xia, J., Liu, H., Wu, Y., Zhang, L., Yu, Z., Fang, M., et al. (2020). Clinical course and outcomes of critically ill patients with SARS-CoV-2 pneumonia in Wuhan, China: a singlecentered, retrospective, observational study. Lancet Respir Med 8, 475481.

Zhou, P., Yang, X.L., Wang, X.G., Hu, B., Zhang, L., Zhang, W., Si, H.R., Zhu, Y., Li, B., Huang, C.L., et al. (2020). A pneumonia outbreak associated with a new coronavirus of probable bat origin. Nature 579, 270-273. 